\title{
E-Cadherin and Angiopoietin-2 as Potential Biomarkers for Colorectal Cancer With Peritoneal Carcinomatosis
}

\author{
HEE KYUNG KIM $^{1 *}$, YAEWON YANG ${ }^{1 *}$, SEONGGYU BYEON ${ }^{1}$, YUSOOK JEONG ${ }^{1}$, JIHYUN KWON ${ }^{1}$, \\ KI HYEONG LEE ${ }^{1,2}$, SEUNG-MYOUNG SON ${ }^{3,4}$ and HYE SOOK HAN ${ }^{1,2}$ \\ ${ }^{1}$ Department of Internal Medicine, Chungbuk National University Hospital, Cheongju, Republic of Korea; \\ ${ }^{2}$ Department of Internal Medicine, Chungbuk National University College of Medicine, Cheongju, Republic of Korea; \\ ${ }^{3}$ Department of Pathology, Chungbuk National University Hospital, Cheongju, Republic of Korea; \\ ${ }^{4}$ Department of Pathology, Chungbuk National University College of Medicine, Cheongju, Republic of Korea
}

\begin{abstract}
Background/Aim: E-Cadherin has been implicated in cell-cell adhesion, and soluble E-cadherin is involved in angiogenesis and resistance to anti-angiogenic therapy in several cancer types. This study aimed to investigate the expression and clinical significance of soluble E-cadherin and other angiogenesis-related factors in plasma and malignant ascites of colorectal cancer (CRC) in patients with peritoneal carcinomatosis (PC). Materials and Methods: Multiplex enzyme-linked immunosorbent assay was performed on 95 body fluid samples (57 plasma and 38 malignant ascites) from patients with CRC. The status of E-cadherin and angiopoietin2 (AGNPT2) was retrospectively evaluated by immunohistochemistry in primary CRC and paired metastatic peritoneal tissues or cell blocks of malignant ascites of 30 patients with peritoneal metastases of CRC. Results: The expression levels of soluble E-cadherin and ANGPT2 in plasma samples were significantly increased in patients with $P C$ compared with those without. E-Cadherin concentration was significantly lower and ANGPT2 concentration was significantly higher in malignant ascites than plasma samples. Expression of E-cadherin was strongly positive, whilst that of ANGPT2 was negative in primary colorectal tissues, metastatic peritoneal tissues, and cell blocks of malignant
\end{abstract}

This article is freely accessible online.

\footnotetext{
*These Authors contributed equally as first authors.

Correspondence to: Hye Sook Han, MD, Ph.D., Department of Internal Medicine, Chungbuk National University College of Medicine, Chungdae-ro 1, Seowon-gu, Cheongju, 28644, Republic of Korea. Tel.: +82 432696359, Fax: +82 432733252, e-mail: sook3529@hanmail.net
}

Key Words: E-Cadherin, angiopoietin-2, colorectal cancer, peritoneal carcinomatosis. ascites by immunohistochemistry. High levels of soluble Ecadherin or ANGPT2 in ascites were negatively associated with overall survival in patients with CRC with malignant ascites. Conclusion: Our findings suggest that soluble Ecadherin and ANGPT2 may be surrogate biomarkers for clinical outcome in patients with PC from CRC.

The survival of patients with metastatic colorectal cancer (CRC) has markedly improved over the past two decades, resulting from the development of combination chemotherapy approaches with molecularly targeted agents such as anti-vascular endothelial growth factor (VEGF-A) or anti-epithelial growth factor receptor $(1,2)$. The US Food and Drug Administration approved bevacizumab, an antibody to VEGF, for first-line chemotherapy based on the survival benefit that was demonstrated in a landmark trial (1). However, many patients with metastatic CRC develop resistance to anticancer treatment or eventual disease progression, and their prognosis is still poor, with a 5-year survival of less than 3 years (3). Patients with CRC with peritoneal metastases generally have a shorter survival than those without peritoneal involvement, and these cases are classified as the M1C category in the eighth edition of the American Joint Committee on Cancer Staging (4). Angiogenesis plays a crucial role in the multistep process of peritoneal carcinomatosis (PC), enabling invasion and migration, and providing oxygen and nutrients (5). Furthermore, increased vascular permeability due to VEGF is considered an important driver of increased production of malignant ascites (6).

E-Cadherin is a $120-\mathrm{kDa}$ transmembrane molecule that maintains the integrity of the conjunct cell-cell structure and influences cellular adherence (7). The epithelialmesenchymal transition (EMT) is involved in cancer cell growth, invasion and migration, and in particular, E-cadherin plays an essential role in cell-cell adhesion (8). Loss of E- 
cadherin has been associated with tumor progression and invasion (9). Soluble E-cadherin also induces angiogenesis and hence can contribute to the resistance to anti-angiogenic therapy (10). Several studies showed that the expression of E-cadherin to be correlated with prognosis in patients with CRC $(11,12)$; loss of E-cadherin in CRC tumor tissues and elevated serum E-cadherin levels were associated with poor prognosis in patients with CRC. Recent studies showed that angiopoietin-2 (ANGPT2) represents a promising biomarker in VEGF-directed anti-angiogenic therapy, and the level of plasma ANGPT2 is associated with poor prognosis in a number of malignancies $(13,14)$. However, little information is available regarding the expression and clinical significance of E-cadherin and angiogenesis-related factors such as VEGF and ANGPT2 in CRC with PC.

In this study, we aimed to evaluate the level of soluble Ecadherin and angiogenesis-related factors in plasma and malignant ascites from patients with $\mathrm{CRC}$ and to assess their prognostic value in survival in patients with PC from CRC.

\section{Patients and Methods}

Patients. Cases were reviewed from a database of patients with pathologically confirmed primary colorectal adenocarcinoma at Chungbuk National University Hospital between May 2012 and September 2019. All patients provided informed consent for use of specimens. Plasma samples classified as early stage (stage I and II), locally advanced stage (stage III), liver-only metastases (stage IVA), and PC (stage IVC) were selected. Malignant ascites samples were selected from a pathological database of patients who had undergone tapping of ascitic fluid and who were diagnosed with primary colorectal adenocarcinoma. Malignant ascites was defined in patients with positive cytology in ascitic fluid; patients with negative ascitic cytology finding were diagnosed on clinical and radiological findings. The radiological parameters of PC from computed tomography were as follows: (i) Ascites, (ii) thickening of bowel walls, (iii) increased density of peritoneal fat, (iv) the presence of peritoneal seeding nodules, or (v) hydronephrosis from ureteral obstruction (15). Patients were excluded from the study if they had any other cancer type or other benign diseases with ascites, such as tuberculosis peritonitis, liver cirrhosis, or end stage renal disease. Clinicopathological data, including age, sex, location of primary tumor, histological classification, stage, timing of PC, and survival, were obtained from medical records and pathology reports.

The study protocol was reviewed and approved by the Institutional Review Board of the Chungbuk National University Hospital, South Korea (IRB approval number: CBNUH 2019-04024), and written informed consent for use of specimens from the National Biobank of Korea was obtained from all study participants.

Enzyme-linked immunosorbent assay. All plasma and ascites samples were obtained from the National Biobank of Korea. Ascites samples were collected at the time of diagnostic or therapeutic paracentesis in patients with CRC with malignant ascites. Samples were centrifuged at $3,200 \times g$ for $10 \mathrm{~min}$ to eliminate large cell particles and cell debris. The supernatant from each sample was then aliquoted into microcentrifuge tubes and stored at $-80^{\circ} \mathrm{C}$ until further analysis. The expression levels of soluble E-cadherin, VEGF, and ANGPT2 were quantified using commercially available enzyme-linked immunosorbent assay kits (Quantikine; R\&D Systems, Minneapolis, MN, USA) according to the manufacturer's instructions. The estimated limits of quantitation for each analyte were $0.31 \mathrm{ng} / \mathrm{ml}$ for E-cadherin, $31.3 \mathrm{pg} / \mathrm{ml}$ for VEGF and 46.9 $\mathrm{pg} / \mathrm{ml}$ for ANGPT2.

Immunohistochemistry (IHC). E-Cadherin and ANGPT2 were evaluated by IHC in surgically resected tissues of primary CRC and peritoneal metastases, as well as cell blocks of malignant ascites with confirmed adenocarcinoma. The cell blocks were prepared by centrifuging the ascitic fluid specimens for $10 \mathrm{~min}$ at $598 \times \mathrm{g}$ ). After the supernatant was discarded, the pellet was resuspended in $95 \%$ ethanol and centrifuged again. The pellet was then cut to a suitable size and transferred to a tissue-embedding cassette. For tissue processing, the cell block of malignant ascites was obtained by subjecting the embedded cell pellet to three changes of alcohol, two changes of xylene, and two changes of paraffin wax. Paired formalin-fixed, paraffin-embedded surgically resected specimens of primary CRC and peritoneal metastatic tissues were selected. Immunohistochemical staining of E-cadherin and ANGPT2 proteins was performed on $4 \mu \mathrm{M}$ paraffin sections of cell blocks and tissue blocks using a BenchMark XT autostainer (Ventana Medical Systems Inc., Tucson, AZ, USA) according to the manufacturer's instructions. Monoclonal antibodies against E-cadherin (EP700Y, 1:200; Cell Marque, Rocklin, CA, USA) and ANGPT2 (sc-74402, 1:50; Santa Cruz Biotechnology, Santa Cruz, CA, USA) were used. Membranous staining of $>50 \%$ of tumor cells was considered positive for E-cadherin. ANGPT2 expression was defined as positive when staining was observed in the cytoplasm in $\geq 10 \%$ of cells in the whole tissue section.

Statistical analysis. The Mann-Whitney $U$-test and Wilcoxon signed-rank test were performed to compare the expression levels of E-cadherin and angiogenesis-related factors of unpaired (plasma $\mathrm{n}=57$, ascites $\mathrm{n}=38$ ) and paired (tissues from primary CRC and peritoneal metastasis $n=30$ ) groups, respectively. The Kaplan-Meier method was used for estimation of overall survival (OS), and differences in survival according to the expression level of Ecadherin and ANGPT2 were analyzed using the log-rank test. The reference ranges for E-cadherin and ANGPT2 in ascites samples were not available; hence, the minimum $p$-value approach (16) was implemented to determine the optimal cut-off for E-cadherin and ANGPT2 for the best separation of patient OS by X-Tile software (17), version 3.6.1 (Yale University, New Haven, CT, USA). X-Tile plots can be applied to divide a population into two levels (low and high level) and provide an "on-the-fly" histogram with an associated Kaplan-Meier curve, and the best $p$-value is available after rigorous statistical evaluation by X-Tile.

Data were analyzed using IBM SPSS software (ver. 25.0; IBM Co., Armonk, NY, USA). All statistical analyses were two-sided tests with significance defined as $p<0.05$.

\section{Results}

Patient characteristics. This study included 57 plasma samples and 38 ascites samples from patients with peritoneal metastases from CRC, as well as 13 paired plasma-ascites 
Table I. Baseline characteristics of patients with colorectal cancer.

\begin{tabular}{|c|c|c|c|c|}
\hline Characteristic & & Plasma $(\mathrm{n}=57)$ & Ascites $(n=38)$ & $\begin{array}{c}\text { Paired primary CRC } \\
\text { and peritoneal } \\
\text { metastasis }(n=30)\end{array}$ \\
\hline Age, years & Median (range) & $65(38-83)$ & $65(38-82)$ & $72(39-85)$ \\
\hline \multirow[t]{2}{*}{ Gender, n (\%) } & Male & $36(63.2)$ & $22(57.9)$ & $15(50.0)$ \\
\hline & Female & $21(36.8)$ & $16(42.1)$ & $15(50.0)$ \\
\hline \multirow[t]{3}{*}{ Location of primary tumor, $\mathrm{n}(\%)$} & Cecum, ascending, transverse & $20(35.1)$ & $12(31.6)$ & $9(30.0)$ \\
\hline & Descending, sigmoid & $24(42.1)$ & $21(55.3)$ & $19(63.3)$ \\
\hline & Rectum & $13(22.8)$ & $5(13.2)$ & $2(6.7)$ \\
\hline \multirow[t]{3}{*}{ Histology, n (\%) } & Adenocarcinoma & $53(93.0)$ & $29(76.3)$ & $28(93.3)$ \\
\hline & Mucinous carcinoma & $1(1.7)$ & $2(5.3)$ & $2(6.7)$ \\
\hline & Unknown & $3(5.3)$ & $7(18.4)$ & 0 \\
\hline \multirow[t]{3}{*}{ Histological grade } & Well/moderate & $50(87.7)$ & $21(55.3)$ & $24(80.0)$ \\
\hline & Poor & $2(3.5)$ & $1(2.6)$ & $4(13.3)$ \\
\hline & Unknown & $5(3.8)$ & $16(42.1)$ & $2(6.7)$ \\
\hline \multirow[t]{4}{*}{ Stage, n $(\%)$} & Early (stage I and II) & $14(24.6)$ & & NA \\
\hline & Locally advanced (stage III) & $14(24.6)$ & & NA \\
\hline & Liver only metastases (stage IVA) & $14(24.6)$ & & NA \\
\hline & Peritoneal metastases (stage IVC) & $15(26.3)$ & $38(100)$ & $30(100)$ \\
\hline \multirow{3}{*}{ Cytology of ascites, n (\%) } & Positive & NA & $15(39.4)$ & NA \\
\hline & Negative & NA & $15(39.4)$ & NA \\
\hline & Indeterminate/unknown & NA & $8(21.2)$ & NA \\
\hline Peritoneal metastases, $\mathrm{n}(\%)$ & Synchronous & NA & $21(55.3)$ & $26(86.7)$ \\
\hline \multirow[t]{2}{*}{ Peritoneal metastasis sample, n (\%) } & Surgically resected tissue & NA & NA & $20(66.7)$ \\
\hline & Cell block of malignant ascites & NA & NA & $10(33.3)$ \\
\hline
\end{tabular}

NA: Not applicable.

samples from the same patients, that were selected between May 2012 and September 2019. We also evaluated formalinfixed, paraffin-embedded tissues of primary tumors and corresponding peritoneal metastatic tissues, as well as ethanol-fixed, paraffin-embedded cell blocks of malignant ascites for 30 patients with peritoneal metastases of CRC. The baseline characteristics of patients overall are shown in Table I. Plasma samples were selected from patients with early stage CRC ( $n=14$, stage I, II), locally advanced stage $(n=14$, stage III), liver-only metastases $(n=14$, stage IVA), and PC $(n=15$, stage IVC). In the ascites sample set, 15 patients $(39.4 \%)$ had positive cytology for metastatic adenocarcinoma and 21 patients $(55.3 \%)$ had synchronous PC when they were initially diagnosed with CRC. Of the 30 samples from patients with PC, $20(66.7 \%)$ were identified in surgically resected peritoneal tissues and $10(33.3 \%)$ in cell blocks of malignant ascites.

Expression of soluble E-cadherin and angiogenesis-related factors. We first analyzed the expression levels of soluble Ecadherin and the angiogenesis-related factors VEGF and ANGPT2 in the 57 plasma samples from patients with CRC (Figure 1, Table II). The expression level of plasma Ecadherin in patients with PC of CRC was not significantly different than the level in patients with early-stage CRC
( $p=0.089$ ) but it was significantly higher than the level in patients with locally advanced CRC $(p=0.002)$ and liver-only metastases ( $p=0.004$ ) (Figure 1A). VEGF was also expressed at a high level in patients with PC compared with early or locally advanced or liver only metastases but there was no statistical significance (Figure 1B). The expression level of ANGPT2 in patients with PC was significantly higher than the level in patients with early-stage CRC ( $p=0.010)$, locally advanced disease $(p=0.001)$ or liver-only metastases $(p=0.021)$ (Figure 1C).

Next, we compared the expression levels of soluble Ecadherin and ANGPT2 in plasma samples and cell-free fluid of malignant ascites from patients with PC. The expression of soluble E-cadherin in cell-free fluid of ascites was significantly lower than that in plasma samples (Figure 2A left panel, Table III, $p<0.0001)$, whilst that of ANGPT2 was significantly higher (Figure 2A right panel, Table III, $p=0.0003)$. In the 13 paired samples of plasma and ascites, the expression of E-cadherin was significantly lower (Figure 2B left panel, $p=0.001)$ and ANGPT2 expression was significantly higher (Figure 2B right panel, $p=0.010$ ) in ascites than in plasma samples.

IHC analysis of E-cadherin and ANGPT2. E-Cadherin and ANGPT2 expression were retrospectively evaluated by IHC 
A

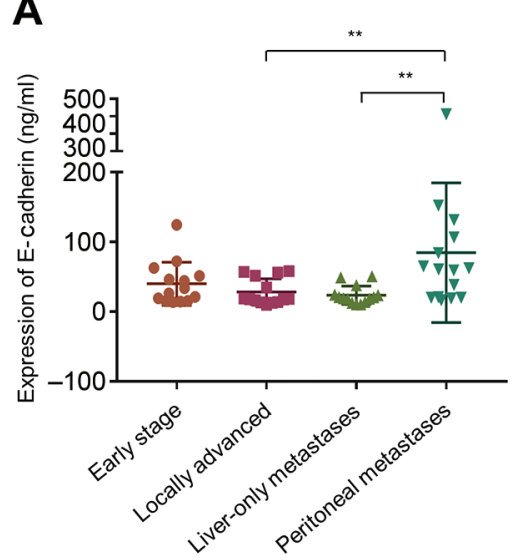

B

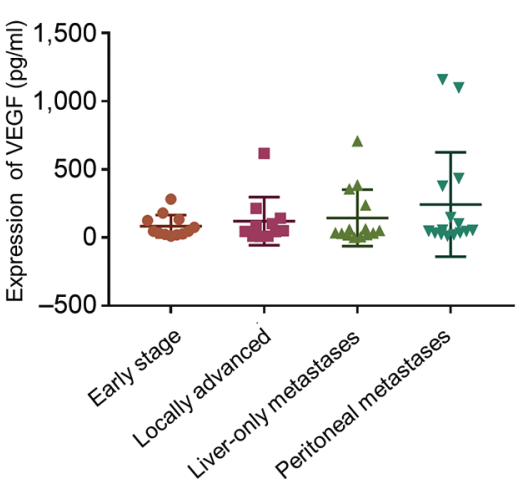

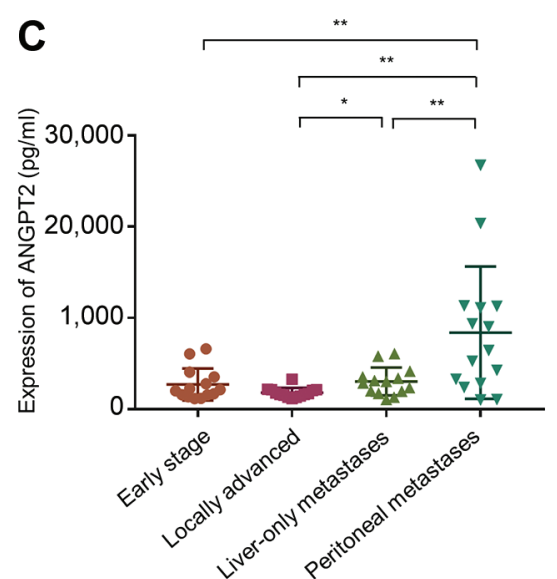

Figure 1. Expression levels of soluble E-cadherin (A), vascular endothelial growth factor (VEGF) (B), and angiopoietin-2 (ANGPT2) (C) in plasma samples according to clinical stage of colorectal cancer. Statistical analyses were performed using the Mann-Whitney U-test. Significantly different at; $* p<0.05$ and $* * p<0.01$.

Table II. Expression (mean \pm standard deviation) of soluble E-cadherin and angiogenesis-related factors in plasma samples.

\begin{tabular}{lcccc}
\hline Factor & $\begin{array}{c}\text { Early stage } \\
(\text { stage I and II) } \\
(\mathrm{n}=14)\end{array}$ & $\begin{array}{c}\text { Locally advanced } \\
(\text { stage III) } \\
(\mathrm{n}=14)\end{array}$ & $\begin{array}{c}\text { Liver only metastases } \\
(\text { stage IVA) } \\
(\mathrm{n}=14)\end{array}$ & $\begin{array}{c}\text { Peritoneal metastases } \\
(\text { stage IVC) } \\
(\mathrm{n}=15)\end{array}$ \\
\hline E-cadherin $(\mathrm{ng} / \mathrm{ml})$ & $40.27 \pm 30.96$ & $29.29 \pm 19.42$ & $23.39 \pm 12.70$ & $84.91 \pm 100.12$ \\
VEGF $(\mathrm{pg} / \mathrm{ml})$ & $84.26 \pm 82.24$ & $123.07 \pm 185.72$ & $141.58 \pm 200.04$ & $243.03 \pm 382.28$ \\
ANGPT2 $(\mathrm{pg} / \mathrm{ml})$ & $2,711.78 \pm 1757.05$ & $1,817.93 \pm 564.87$ & $2,938.86 \pm 1506.84$ & $8,388.87 \pm 7257.79$ \\
\hline
\end{tabular}

ANGPT2: Angiopoietin-2; VEGF: vascular endothelial growth factor.

in primary CRC tissues and paired pathologically confirmed surgically-resected peritoneal metastatic tissues, as well as cell blocks of malignant ascites of 30 patients with peritoneal CRC involvement. E-Cadherin expression was strongly positive in all primary $\mathrm{CRC}$ tissues, peritoneal metastatic tissues and cell blocks of malignant ascites (Figure 3A-C). ANGPT2 was not expressed in primary CRC tissues, peritoneal metastatic tissues or cell blocks of malignant ascites (Figure 3D-F).

Survival analysis. We assessed the OS of patients with with PC from CRC according to the expression level of soluble E-cadherin and ANGPT2 in ascites samples. According to the minimum $p$-value approach, $\mathrm{X}$-tile software was used to determine the optimal cut-off level of soluble E-cadherin and angiogenesis-related factors in ascites. The minimum $p$-value (0.00006) was when the level of soluble E-cadherin was 1.21 $\mathrm{ng} / \mathrm{ml}$, while it was 0.27332 for ANGPT2 at $13.3 \mathrm{ng} / \mathrm{ml}$. Kaplan-Meier analysis for OS with these cut-off points demonstrated that high soluble E-cadherin in malignant ascites was correlated with shorter OS (median: 28.9 vs. 17.5 months in the groups with low vs. high soluble E-cadherin level; $p=0.023$, Figure 4A). A high level of soluble ANGPT2 in malignant ascites also tended to be associated with poor OS but there was no statistically significant difference (median: 28.1 vs. 17.5 months in groups with low vs. high ANGPT2 level; $p=0.344$, Figure 4B).

\section{Discussion}

In the current study, we evaluated the expression of soluble E-cadherin and the angiogenesis-related factors VEGF and ANGPT2 in plasma and ascites samples from patients with CRC. To the best of our knowledge, this is the first report on the expression of soluble E-cadherin and ANGPT2 in plasma and malignant ascites from patients with PC from CRC. The levels of soluble E-cadherin and ANGPT2 were elevated in the plasma of patient with PC from CRC compared with those with early or locally advanced or metastatic liver disease. High soluble E-cadherin expression has been associated with poor prognosis in multiple cancer types in addition to CRC $(18,19)$, including prostate cancer (20), non-small-cell lung cancer (21), and melanoma (22). Overexpression of ANGPT2 is also correlated with tumor 

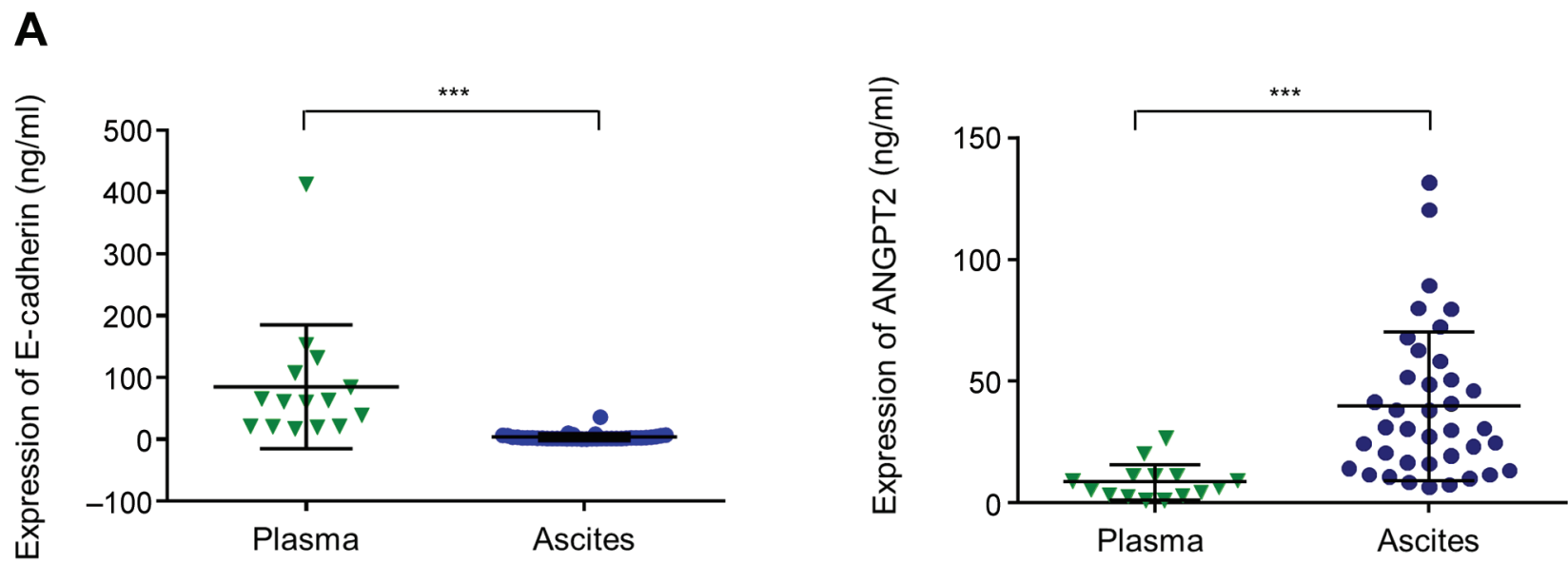

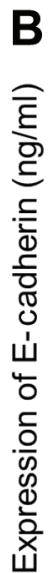
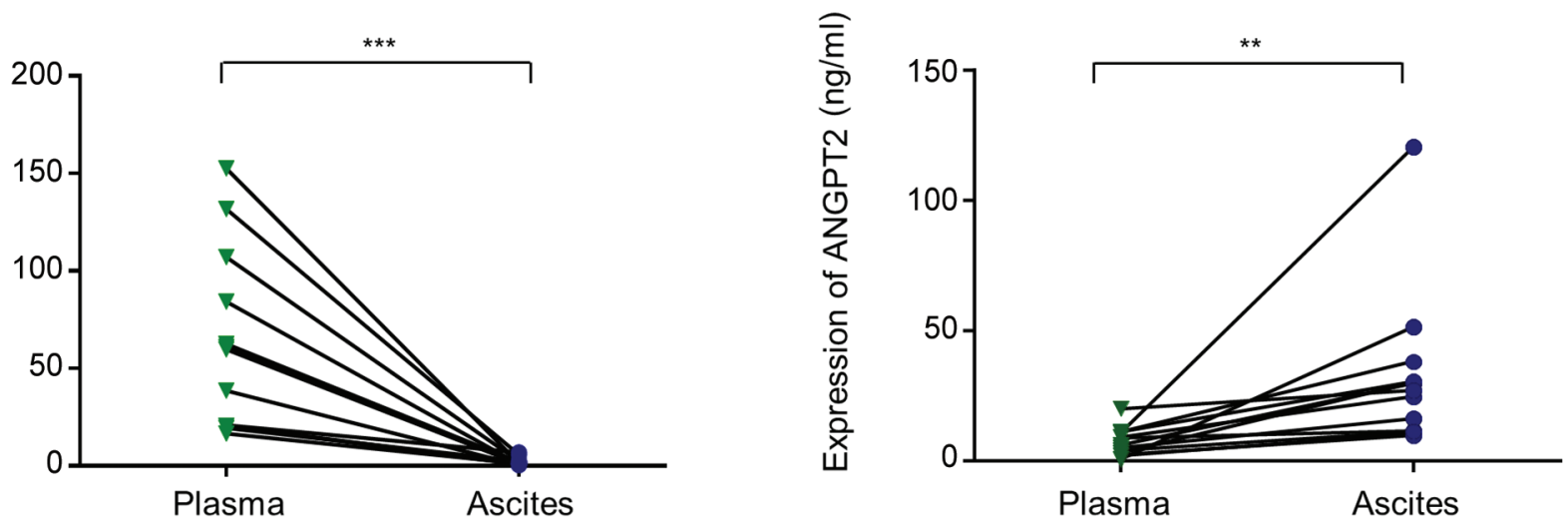

Figure 2. Expression levels of soluble E-cadherin (left) and angiopoietin-2 (ANGPT2) (right) in individual plasma and ascites samples (A) and in paired plasma and ascites samples $(B)$ from patients with peritoneal carcinomatosis from colorectal cancer. Statistical analyses were performed using the Mann-Whitney U-test and Wilcoxon signed-rank test. Significantly different at: **p<0.01 and ***p<0.001.

angiogenesis and progression in various types of cancer such as melanoma, glioblastoma, breast cancer, renal cell carcinoma and CRC (23-25). Moreover, soluble E-cadherin might be an angiogenic regulator responsible for resistance to anti-VEGF treatments (10) and a high serum ANGPT2 level was associated with poor response to anti-VEGFcontaining treatment in patients with CRC (26). In our study, the difference in plasma expression of E-cadherin and ANGPT2 was greatest between patients with PC and those with liver-only metastases, indicating that high expression of E-cadherin and ANGPT2 may be specific biomarkers for PC in patients with CRC.

PC with malignant ascites in CRC has a dismal prognosis, with survival less than 1 year following diagnosis (27). In addition, patients with malignant ascites experience significant symptoms and a poor quality of life. The present study suggests that high levels of soluble E-cadherin and ANGPT2 in ascites samples was associated with poor
Table III. Expression levels (mean \pm standard deviation) of soluble Ecadherin and angiopoietin-2 (ANGPT2) in samples of plasma and ascites from patients with colorectal cancer with peritoneal carcinomatosis.

\begin{tabular}{lccr}
\hline & \multicolumn{3}{c}{ Expression level } \\
\cline { 2 - 4 } Factor & Plasma $(\mathrm{n}=15)$ & Ascites $(\mathrm{n}=38)$ & $p$-Value \\
\hline E-cadherin $(\mathrm{ng} / \mathrm{ml})$ & $84.91 \pm 100.12$ & $3.60 \pm 5.86$ & $<0.0001$ \\
ANGPT2 $(\mathrm{pg} / \mathrm{ml})$ & $8,388.87 \pm 7257.79$ & $39,668.57 \pm 30,626.22$ & 0.0003 \\
\hline
\end{tabular}

survival in patients with CRC with malignant ascites. Therefore, soluble E-cadherin and ANGPT2 might be useful prognostic biomarkers and therapeutic targets in patients with PC from CRC who have grave prognosis and poor response to systemic therapy. 

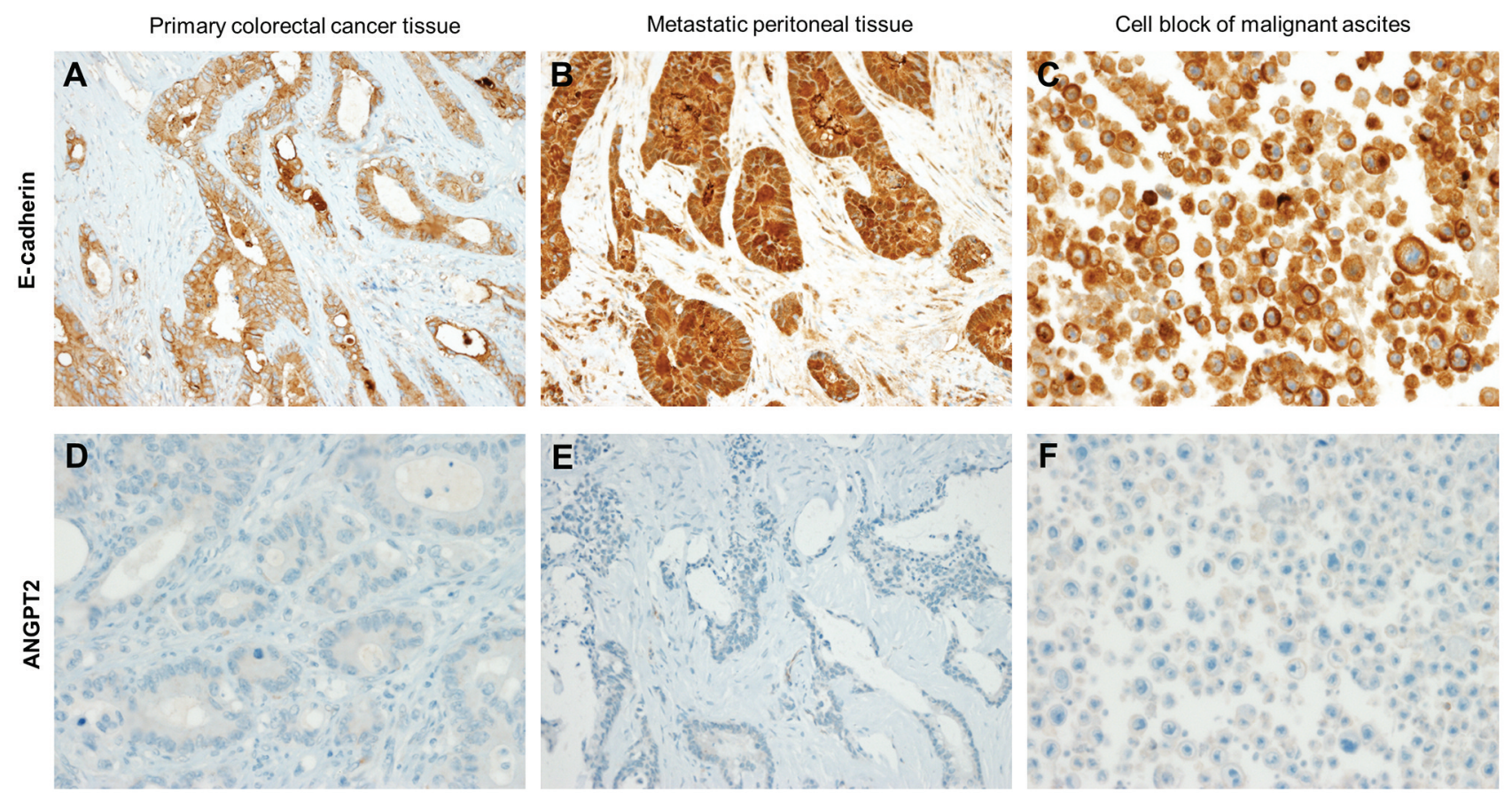

Figure 3. Representative immunohistochemical staining results for E-cadherin and angiopoietin-2 expression in patients with colorectal cancer with peritoneal involvement. E-Cadherin was strongly expressed in primary colorectal cancer tissue (A; original magnification, $\times 200)$, peritoneal metastatic tissues $(B$; original magnification, $\times 200)$ and cell block of malignant ascites $(C$; original magnification, $\times 400)$. Angipoietin-2 was not expressed in primary colorectal cancer tissue (D; original magnification, $\times 200)$, metastatic peritoneal tissues $(E ;$ original magnification, $\times 200)$ or cell block of malignant ascites ( $F$; original magnification, $\times 400)$.

A

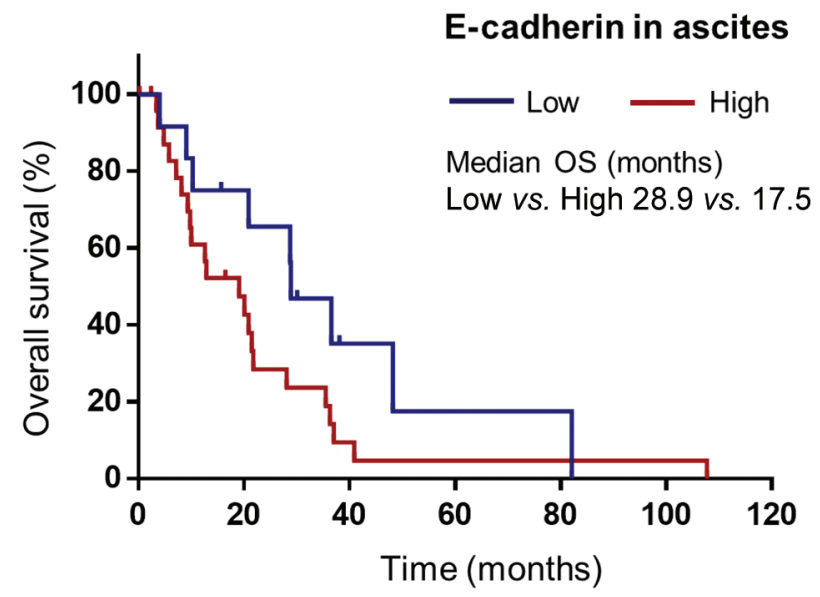

B

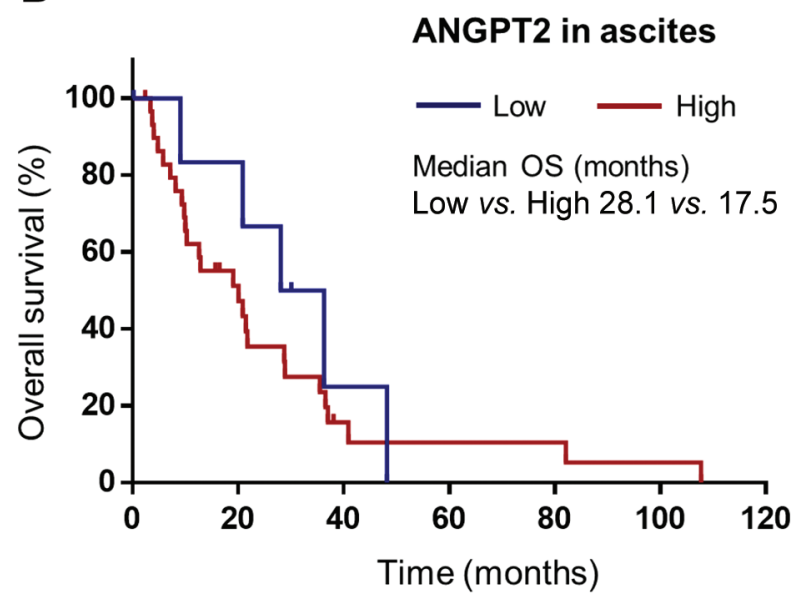

Figure 4. Overall survival according to the levels of soluble E-cadherin (A) and angiopoietin-2 (B) in malignant ascites in patients with colorectal cancer with peritoneal carcinomatosis. Statistical analysis was performed using the Kaplan-Meier method.

We also performed IHC analysis of E-cadherin and ANGPT2 in primary CRC tissues and paired peritoneal metastatic tumor tissues as well as cell block of malignant ascites. E-Cadherin expression was very high, not only in primary CRC tissues but also in peritoneal metastases or cell blocks of malignant ascites, but it was expressed at low levels in cell-free fluid of malignant ascites. In contrast, ANGPT2 was rarely expressed in primary $\mathrm{CRC}$, peritoneal metastatic tissues and cell blocks of malignant 
ascites, while high expression was detected in cell-free fluid of malignant ascites. One potential explanation for the discrepancy in E-cadherin expression between malignant cells in tumor tissues and cell blocks of malignant ascites with levels in cellfree fluid of malignant ascites might be from the complex metastasis mechanisms related to E-cadherin (26). Loss of Ecadherin is associated with increased EMT and local invasion $(28,29)$, and this loss was thought to be followed by an increased number of circulating tumor cells and extravasation at a secondary site where tumor cells establish metastases. However, a recent study showed that although loss of Ecadherin is indeed associated with increased local invasion, it is also associated with reduced metastasis, a finding that supports E-cadherin as an experimental target to prevent metastasis $(30,31)$. Padmanaban et al. observed that E-cadherin is required for metastasis for breast cancer and is expressed by metastases as well as primary tumors (32). A discordance between the IHC results for ANGPT2 and the level of ANGPT2 in ascites was also observed, showing that ANGPT2 IHC was negative whilst high expression of ANGPT2 was detected in cell-free fluid in malignant ascites. Consistent with this result, other studies reported that up-regulation of ANGPT2 reduced tumor growth and metastasis $(33,34)$ as ANPGT2 can induce tumor apoptosis by transient exacerbation of tumor hypoxia (35). There are several questions to be answered related to Ecadherin, ANGPT2 and tumor angiogenesis, and further studies are warranted to elucidate the underlying mechanism of action for the effects of E-cadherin and ANGPT2 expression on cancer progression or metastasis.

The present study has some limitations. Firstly, this study was a retrospective analysis with a limited number of plasma and ascites samples from patients with CRC, and we were unable to evaluate angiogenesis, inflammation and EMT-related markers. Secondly, we were also unable to evaluate the prognostic value of soluble E-cadherin and ANGPT2 in plasma samples or the predictive value for anti-VEGF therapy because of the limited number of plasma samples from patients with PC.

In conclusion, our results suggest that soluble E-cadherin and ANGPT2 are expressed in plasma and ascites samples in patients with PC from CRC, and high levels of soluble Ecadherin and ANGPT2 in malignant ascites were associated with poor prognosis. Our results suggest that soluble Ecadherin and ANGPT2 might be surrogate markers for clinical outcome in CRC with PC.

\section{Conflicts of Interest}

The Authors have no conflicts of interest to declare.

\section{Authors' Contributions}

Acquisition of data, analysis of data, interpretation of data, drafting of article, proofreading and approval of the article: HKK, YY, and
SMS. Interpretation of data, drafting and final approval of article: SB, YJ, JK and KHL. Conception and design of study, analysis of data, interpretation of data, drafting and final approval of article: SMS and HSH

\section{Acknowledgements}

This work was supported by the research grant of the Chungbuk National University Hospital in 2019. All plasma and ascites samples in this study were provided by the Biobank of Chungbuk National University Hospital, which is supported by the Ministry of Health, Welfare and Family Affairs.

\section{References}

1 Hurwitz H, Fehrenbacher L, Novotny W, Cartwright T, Hainsworth J, Heim W, Berlin J, Baron A, Griffing S, Holmgren E, Ferrara N, Fyfe G, Rogers B, Ross R and Kabbinavar F: Bevacizumab plus irinotecan, fluorouracil, and leucovorin for metastatic colorectal cancer. N Engl J Med 350(23): 2335-2342, 2004. PMID: 15175435 . DOI: 10.1056/NEJMoa032691

2 Van Cutsem E, Köhne CH, Hitre E, Zaluski J, Chang Chien CR, Makhson A, D’Haens G, Pintér T, Lim R, Bodoky G, Roh JK, Folprecht G, Ruff P, Stroh C, Tejpar S, Schlichting M, Nippgen J and Rougier P: Cetuximab and chemotherapy as initial treatment for metastatic colorectal cancer. N Engl J Med 360(14): 14081417, 2009. PMID: 19339720. DOI: 10.1056/NEJMoa0805019

3 Carbone C, Piro G, Simionato F, Ligorio F, Cremolini C, Loupakis F, Alì G, Rossini D, Merz V, Santoro R, Zecchetto C, Zanotto M, Di Nicolantonio F, Bardelli A, Fontanini G, Tortora $\mathrm{G}$ and Melisi D: Homeobox B9 mediates resistance to antiVEGF therapy in colorectal cancer patients. Clin Cancer Res 23(15): 4312-4322, 2017. PMID: 28298545. DOI: 10.1158/ 1078-0432.CCR-16-3153

4 Weiser MR: AJCC 8th Edition: Colorectal Cancer. Ann Surg Oncol 25(6): 1454-1455, 2018. PMID: 29616422. DOI: 10.1245/ s10434-018-6462-1

5 Rmali KA, Puntis MC and Jiang WG: Tumour-associated angiogenesis in human colorectal cancer. Colorectal Dis 9(1): 3-14, 2007. PMID: 17181841. DOI: 10.1111/j.1463-1318.2006.01089.x

6 Tamsma JT, Keizer HJ and Meinders AE: Pathogenesis of malignant ascites: Starling's law of capillary hemodynamics revisited. Ann Oncol 12(10): 1353-1357, 2001. PMID: 11762804. DOI: 10.1023/a:1012504904713

7 Wheelock MJ, Buck CA, Bechtol KB and Damsky CH: Soluble 80-kd fragment of cell-CAM 120/80 disrupts cell-cell adhesion. J Cell Biochem 34(3): 187-202, 1987. PMID: 3611200. DOI: $10.1002 /$ jcb. 240340305

8 Jeanes A, Gottardi CJ and Yap AS: Cadherins and cancer: how does cadherin dysfunction promote tumor progression? Oncogene 27(55): 6920-6929, 2008. PMID: 19029934. DOI: 10.1038/onc.2008.343

9 Canel M, Serrels A, Frame MC and Brunton VG: E-cadherinintegrin crosstalk in cancer invasion and metastasis. J Cell Sci 126(Pt 2): 393-401, 2013. PMID: 23525005. DOI: 10.1242/jcs.100115

10 Tang MKS, Yue PYK, Ip PP, Huang RL, Lai HC, Cheung ANY, Tse KY, Ngan HYS and Wong AST: Soluble E-cadherin promotes tumor angiogenesis and localizes to exosome surface. Nat Commun 9(1): 2270, 2018. PMID: 29891938. DOI: $10.1038 / \mathrm{s} 41467-018-04695-7$ 
11 Wang R, Ma X, Li Y, He Y, Huang D, Cai S and Peng J: The characteristics and prognostic effect of E-cadherin expression in colorectal signet ring cell carcinoma. PLoS One 11(8): e0160527, 2016. PMID: 27509205. DOI: 10.1371/journal.pone.0160527

12 Okugawa Y, Toiyama Y, Inoue Y, Iwata T, Fujikawa H, Saigusa S, Konishi N, Tanaka K, Uchida K and Kusunoki M: Clinical significance of serum soluble E-cadherin in colorectal carcinoma. J Surg Res 175(2): e67-e73, 2012. PMID: 22277332. DOI: 10.1016/j.jss.2011.11.009

13 Leong A and Kim M: The angiopoietin-2 and TIE pathway as a therapeutic target for enhancing antiangiogenic therapy and immunotherapy in patients with advanced cancer. Int J Mol Sci 21(22): 8689, 2020. PMID: 33217955. DOI: 10.3390/ijms21228689

14 Gillen J, Richardson D and Moore K: Angiopoietin-1 and angiopoietin-2 inhibitors: Clinical development. Curr Oncol Rep 21(3): 22, 2019. PMID: 30806847. DOI: 10.1007/s11912-0190771-9

15 Yajima K, Kanda T, Ohashi M, Wakai T, Nakagawa S, Sasamoto $\mathrm{R}$ and Hatakeyama K: Clinical and diagnostic significance of preoperative computed tomography findings of ascites in patients with advanced gastric cancer. Am J Surg 192(2): 185-190, 2006. PMID: 16860627. DOI: 10.1016/j.amjsurg.2006.05.007

16 Altman D, Lausen B, Sauerbrei W and Schumacher M: Dangers of using "optimal" cutpoints in the evaluation of prognostic factors. JNCI Journal of the National Cancer Institute $86(11)$ : 829-835, 2017. DOI: 10.1093/jnci/86.11.829

17 Camp RL, Dolled-Filhart M and Rimm DL: X-tile: a new bioinformatics tool for biomarker assessment and outcome-based cut-point optimization. Clin Cancer Res 10(21): 7252-7259, 2004. PMID: 15534099. DOI: 10.1158/1078-0432.CCR-04-0713

18 Wilmanns C, Grossmann J, Steinhauer S, Manthey G, Weinhold B, Schmitt-Gräff A and von Specht BU: Soluble serum Ecadherin as a marker of tumour progression in colorectal cancer patients. Clin Exp Metastasis 21(1): 75-78, 2004. PMID: 15065605. DOI: 10.1023/b:clin.0000017204.38807.22

19 Burandt E, Lübbersmeyer F, Gorbokon N, Büscheck F, Luebke AM, Menz A, Kluth M, Hube-Magg C, Hinsch A, Höflmayer D, Weidemann S, Fraune C, Möller K, Jacobsen F, Lebok P, Clauditz TS, Sauter G, Simon R, Uhlig R, Wilczak W, Steurer S, Minner S, Krech R, Dum D, Krech T, Marx AH and Bernreuther C: E-Cadherin expression in human tumors: a tissue microarray study on 10,851 tumors. Biomark Res 9(1): 44, 2021. PMID: 34090526. DOI: 10.1186/s40364-021-00299-4

20 Kuefer R, Hofer MD, Gschwend JE, Pienta KJ, Sanda MG, Chinnaiyan AM, Rubin MA and Day ML: The role of an $80 \mathrm{kDa}$ fragment of E-cadherin in the metastatic progression of prostate cancer. Clin Cancer Res 9(17): 6447-6452, 2003. PMID: 14695147.

21 Charalabopoulos K, Gogali A, Dalavaga Y, Daskalopoulos G, Vassiliou M, Bablekos G, Karakosta A and Constantopoulos S: The clinical significance of soluble E-cadherin in nonsmall cell lung cancer. Exp Oncol 28(1): 83-85, 2006. PMID: 16614715.

22 Billion K, Ibrahim H, Mauch C and Niessen CM: Increased soluble E-cadherin in melanoma patients. Skin Pharmacol Physiol 19(2): 65-70, 2006. PMID: 16685144. DOI: 10.1159/000091972

23 Wang HL, Deng CS, Lin J, Pan DY, Zou ZY and Zhou XY: Expression of angiopoietin-2 is correlated with vascularization and tumor size in human colorectal adenocarcinoma. Tohoku J Exp Med 213(1): 33-40, 2007. PMID: 17785951. DOI: 10.1620/tjem.213.33

24 Kloepper J, Riedemann L, Amoozgar Z, Seano G, Susek K, Yu V, Dalvie N, Amelung RL, Datta M, Song JW, Askoxylakis V, Taylor
JW, Lu-Emerson C, Batista A, Kirkpatrick ND, Jung K, Snuderl M, Muzikansky A, Stubenrauch KG, Krieter O, Wakimoto H, Xu L, Munn LL, Duda DG, Fukumura D, Batchelor TT and Jain RK: Ang-2/VEGF bispecific antibody reprograms macrophages and resident microglia to anti-tumor phenotype and prolongs glioblastoma survival. Proc Natl Acad Sci U.S.A. 113(16): 44764481, 2016. PMID: 27044098. DOI: 10.1073/pnas.1525360113

25 Corso G, Figueiredo J, De Angelis SP, Corso F, Girardi A, Pereira J, Seruca R, Bonanni B, Carneiro P, Pravettoni G, Guerini Rocco E, Veronesi P, Montagna G, Sacchini V and Gandini S: Ecadherin deregulation in breast cancer. J Cell Mol Med 24(11): 5930-5936, 2020. PMID: 32301282. DOI: 10.1111/jcmm.15140

26 Goede V, Coutelle O, Neuneier J, Reinacher-Schick A, Schnell R, Koslowsky TC, Weihrauch MR, Cremer B, Kashkar H, Odenthal M, Augustin HG, Schmiegel W, Hallek M and Hacker UT: Identification of serum angiopoietin-2 as a biomarker for clinical outcome of colorectal cancer patients treated with bevacizumab-containing therapy. Br J Cancer 103(9): 14071414, 2010. PMID: 20924372. DOI: 10.1038/sj.bjc.6605925

27 Lemmens VE, Klaver YL, Verwaal VJ, Rutten HJ, Coebergh JW and de Hingh IH: Predictors and survival of synchronous peritoneal carcinomatosis of colorectal origin: a populationbased study. Int J Cancer 128(11): 2717-2725, 2011. PMID: 20715167. DOI: $10.1002 / \mathrm{ijc} .25596$

28 O'Driscoll L: When E-cadherin becomes unstuck in cancer. $\mathrm{N}$ Engl J Med 383(9): 871-873, 2020. PMID: 32846069. DOI: 10.1056/NEJMcibr2018713

29 Sommariva M and Gagliano N: E-cadherin in pancreatic ductal adenocarcinoma: A multifaceted actor during EMT. Cells 9(4): 1040, 2020. PMID: 32331358. DOI: 10.3390/cells9041040

$30 \mathrm{Na}$ TY, Schecterson L, Mendonsa AM and Gumbiner BM: The functional activity of E-cadherin controls tumor cell metastasis at multiple steps. Proc Natl Acad Sci U.S.A. 117(11): 5931-5937, 2020. PMID: 32127478. DOI: 10.1073/pnas.1918167117

31 Skarkova V, Skarka A, Manethova M, Stefanidi AA and Rudolf E: Silencing of E-cadherin expression leads to increased chemosensitivity to irinotecan and oxaliplatin in colorectal cancer cell lines. Hum Exp Toxicol: 9603271211021479, 2021. PMID: 34075792. DOI: 10.1177/09603271211021479

32 Padmanaban V, Krol I, Suhail Y, Szczerba BM, Aceto N, Bader JS and Ewald AJ: E-cadherin is required for metastasis in multiple models of breast cancer. Nature 573(7774): 439-444, 2019. PMID: 31485072. DOI: 10.1038/s41586-019-1526-3

33 Shim WS, Ho IA and Wong PE: Angiopoietin: a TIE(d) balance in tumor angiogenesis. Mol Cancer Res 5(7): 655-665, 2007. PMID: 17634421. DOI: 10.1158/1541-7786.MCR-07-0072

34 Tait CR and Jones PF: Angiopoietins in tumours: the angiogenic switch. J Pathol 204(1): 1-10, 2004. PMID: 15307132. DOI: 10.1002/path.1618

35 Cao Y, Sonveaux P, Liu S, Zhao Y, Mi J, Clary BM, Li CY, Kontos CD and Dewhirst MW: Systemic overexpression of angiopoietin-2 promotes tumor microvessel regression and inhibits angiogenesis and tumor growth. Cancer Res 67(8): 3835-3844, 2007. PMID: 17440098. DOI: 10.1158/00085472.CAN-06-4056

Received May 17, 2021

Revised July 8, 2021

Accepted July 9, 2021 